\title{
LEEM and PEEM as Probing Tools to Address Questions in Catalysis
}

\author{
Mauricio J. Prieto $^{1} \cdot$ Thomas Schmidt $^{1}$
}

Received: 22 July 2017 / Accepted: 27 July 2017 / Published online: 14 August 2017

(C) The Author(s) 2017. This article is an open access publication

\begin{abstract}
Catalysis is a hot topic in research with the focus on finding catalysts that show better activity or selectivity on processes on technological or industrial interest. The use of model systems of applicable materials has proven to be a successful approach in the last decades to obtain information on the fundamental properties of these materials, leading eventually to a better understanding how real catalysts work. This knowledge is extremely important in the sense that it allows an optimization of the catalyst composition, thus leading to a rational design of new materials. For these fundamental studies, a variety of probing techniques such as X-ray photo-electron spectroscopy, scanning tunnel microscopy, and temperature programmed desorption have been applied. In this article, we discuss how low energy electron microscopy (LEEM) and photoemission electron microscopy (PEEM) can contribute to the fundamental understanding of relevant surface processes taking place on model catalysts. Also, the capability of these techniques on addressing open questions in catalysis is discussed.
\end{abstract}

Mauricio J. Prieto

prieto@fhi-berlin.mpg.de

1 Department of Chemical Physics, Fritz-Haber-Institute of the Max-Planck Society, Faradayweg 4-6, 14195 Berlin, Germany

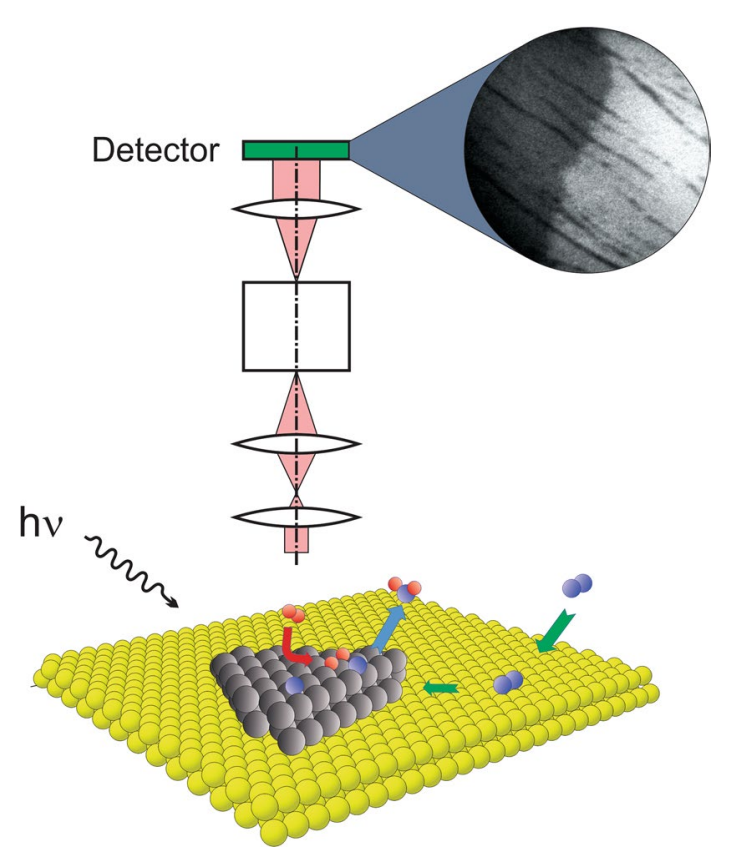

Keywords LEEM $\cdot$ PEEM < microscopy $\cdot$ Spectroscopy and general characterisation $\cdot$ Model systems $\cdot$ Catalysis

\section{Introduction}

The phenomenon of catalysis has been the subject of extensive research for many decades in different fields and in all its forms such as heterogeneous, homogeneous and electrochemical [1-4]. Its importance relies on the fact that, in the presence of catalysts, many chemical processes that in principle require extreme thermal and pressure conditions to occur can be conducted under milder conditions for different 
purposes, ranging from clean energy production to the synthesis of new compounds with interesting mechanical or chemical properties [5-7].

Catalysts are complex materials with particular properties that cause a chemical reaction to proceed with a lower activation energy, by allowing the chemical reaction to take an alternative reaction path, thus increasing the kinetics and, in some cases, the selectivity. However, a complete chemical reaction is a collection of different fundamental steps, all happening at the surface of the catalyst. For instance, adsorption, diffusion, bond splitting, bond formation and desorption constitute the most common steps in the majority of chemical reactions on surfaces [8]. For this reason, the so-called active sites are the most important places of the material, where all the action occurs. In general, active sites are surface sites with a specific atomic arrangement or composition (in the case of multicomponent systems) that are involved in individual steps of the catalysed chemical reactions [9]. The importance of these special sites has been the subject of many publications and is widely recognized by the scientific community $[10,11]$. For instance, the higher reactivity of under coordinated atoms on a surface has been investigated by many techniques $[12,13]$. Also, when multicomponent systems are considered, the difference in activity can emerge in a synergistic manner giving the constituents combination a unique property that is not present in any of the individual materials (see e.g. [14]).

In this regard, fundamental studies on catalyst materials using model systems enable the decrease of the degree of complexity of a real catalyst and allow the development of fundamental concepts. In contrast to many other surface sensitive techniques, this approach has proven to be valuable because, in most cases, it is not possible to get valuable information on the surface properties of real catalysts under in operando conditions. However, the concepts forged with this approach of model systems allow the rationalization and the optimization of new materials.

In this article, we present and discuss the potential of low energy electron microscopy (LEEM) and photoemission electron microscopy (PEEM) as complementary tools for addressing fundamental questions in the area of catalysis. The LEEM/PEEM approach to model systems in catalysis is potentially significant due to many aspects. First of all, the technique usually offers the possibility to perform experiments and follow processes in real time and under in situ and in operando conditions. This advantage is useful in the catalysis field because it allows in principle to address changes and issues that are intrinsically dynamic in nature. For example, catalysis is a surface phenomenon, i.e., all processes occur at the topmost atomic layers of a material that is chemically active for a given reaction. Moreover, many processes are involved during the catalysis of a chemical reaction, namely: adsorption, diffusion, recombination, dissociation, bond formation, etc. In addition to the dynamics of the reaction itself, the surface dynamics of the material should be taken into account. For instance, many catalysts are multicomponent materials, having fixed components of different chemical nature such as an active phase (responsible for catalysing the chemical reaction) and a supporting phase (on which the active phase can be dispersed). During a chemical reaction under in operando conditions, the composition, structure and/or the degree of dispersion of the active phase on the support can change and consequently modify the activity of the whole material and hence, these are important aspects that need to be taken into account when transferring concepts from model catalysts to applied systems.

\section{Photoemission Electron Microscopy (PEEM) and Low Energy Electron Microscopy (LEEM)}

A detailed description of the techniques is outside the scope of this article. However, it might be useful to present here some basics of both techniques that could allow the understanding of their potential application to the study of relevant systems in catalysis.

Photoemission electron microscopy is an approved surface science microscopy technique developed by Ernst Brüche in the early 1930s [15] and later on improved by the progress in electron optics (see e.g. ref. [16]). In modern PEEMs, the sample is illuminated with light (UV-lamp or $\mathrm{X}$-rays from a synchrotron source) and the electrons photoemitted from the surface are used for imaging. These electrons are used for imaging by using a particular set-up of lenses (electrostatic or magnetic), also including deflectors and stigmators in combination with an aperture that limits the emittance angle at the sample. Figure 1 shows a general scheme of a typical photoemission electron microscope arrangement. Because of the low kinetic energy, the detected photoemitted electrons are generated in the topmost atomic layers of the sample and therefore, PEEM is inherently a surface sensitive probe. The opportunity of using an energy filtering device for the photoelectrons and a tunable $\mathrm{X}$-ray source (synchrotron radiation) makes it possible to selectively work with electrons having kinetic energies at the minimum of the inelastic mean free path curve, meaning a surface sensitivity of only $0.5 \mathrm{~nm}$. Another interesting property of PEEM using synchrotron radiation is that surface elemental distribution maps of the sample can be obtained by using element specific core level photoemission lines. In this case, image contrast is generated by differences in the chemical composition of the sample, instead of work function differences as in the case of UV-PEEM.

On the other hand, low energy electron microscopy LEEM is an imaging technique developed by Ernst Bauer 


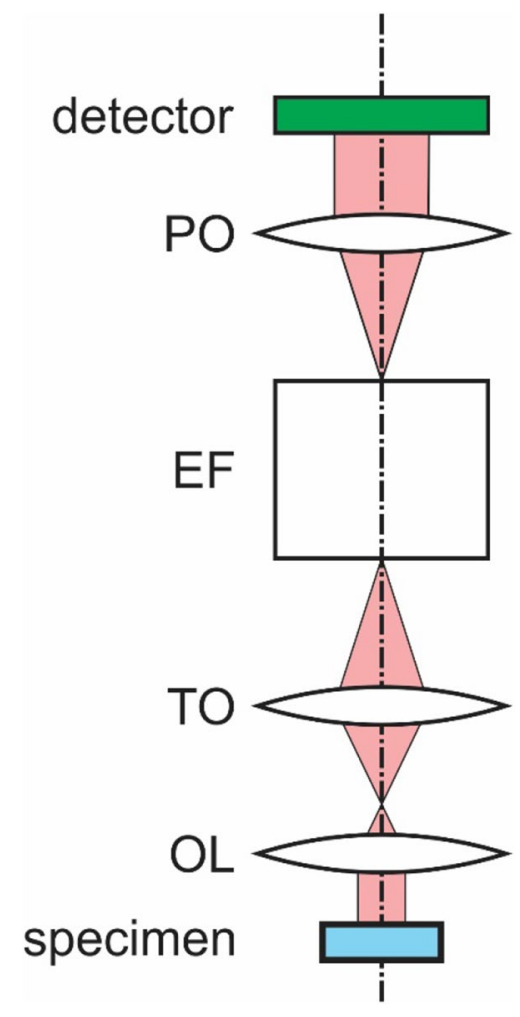

Fig. 1 Schematic set up of a photoemission electron microscope. Elements depicted correspond to objective lens (OL), transfer optics (TO), energy filter (EF) and projection optics (PO)

$[17,18]$. Instead of using electrons for imaging that are photoemitted at the sample surface, in LEEM, a beam of electrons generated in an electron gun illuminates the sample surface, is backscattered and then imaged onto the detector. Typically, the kinetic energy of the electrons at the sample surface is in the $1-100 \mathrm{eV}$ range and after reflexion they are accelerated to about $10-20 \mathrm{keV}$ in the imaging optics column. The main components of a LEEM instrument, as shown in the scheme in Fig. 2, are comparable to those in a PEEM instrument, with the exception of a beam splitting device (BS) with the attached electron gun and an optional electrostatic mirror unit (MO) that enables the correction of spherical and chromatic aberrations and therefore improves the lateral resolution and transmission [19, 20]. In contrast to PEEM, which is a chemical sensitive technique, especially when using X-rays for excitation, LEEM is mainly a structural sensitive method. However, a variety of contrast mechanisms (e.g. diffraction, work function and phase contrast) can lead to a thorough characterization of the sample surface. In addition to microscopy measurements, electron diffraction experiments (LEED) can be performed in LEEM instruments, thus allowing bright and dark field imaging to determine possible rotational domains in complex samples. As stated before, a detailed explanation of the operation principle of PEEM and LEEM is beyond the scope of

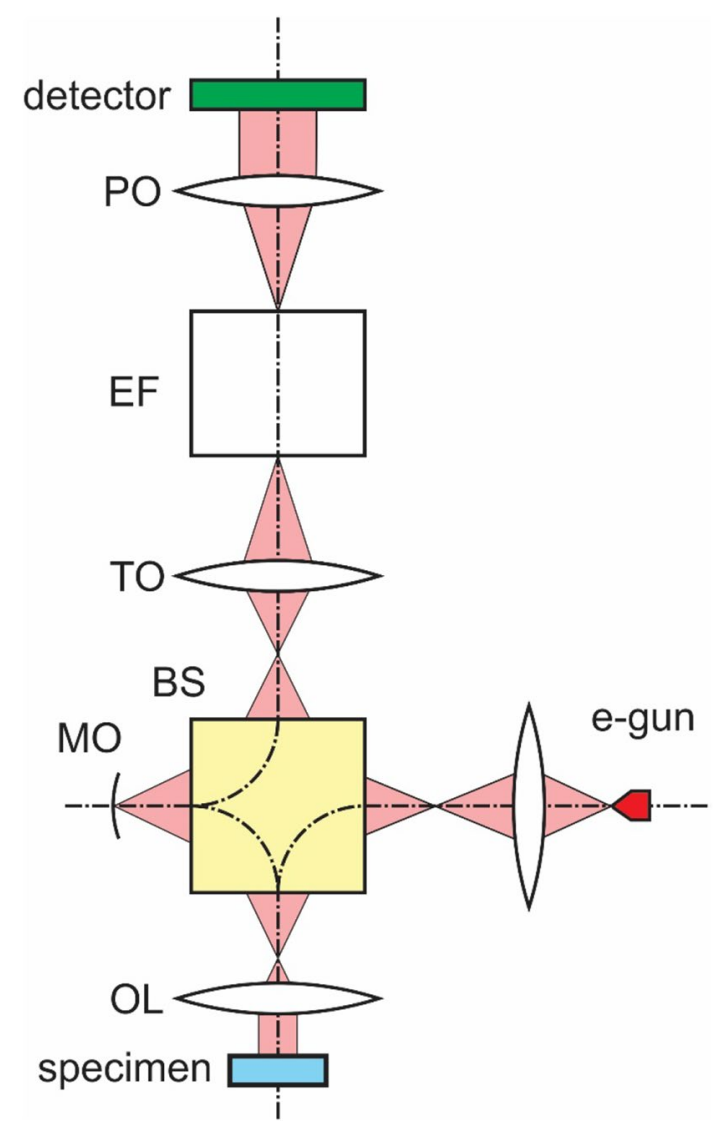

Fig. 2 Schematic set-up of a low energy electron microscope. Elements depicted correspond to objective lens (OL), mirror optics (MO), beam splitter (BS), transfer optics (TO), energy filter (EF) and projection optics (PO)

this article and for this reason the readers are encourage to review the literature addressing the last developments on both LEEM and PEEM (see e.g. [18, 21, 22]).

\section{Application in Catalysis}

One of the main advantages of both LEEM and PEEM as mentioned before is the possibility of following structural and chemical changes on the surface of model catalysts in real time and under well-defined conditions when chemical reactions take place. One clear example is the pioneering work of Jakubith [23] and Rotermund [24] et al. on the evolution of spatio-temporal patterns formed on Pt surfaces during the oxidation of carbon monoxide. On Pt surfaces the reaction proceeds via the recombination of $\mathrm{CO}$ with $\mathrm{O}$ previously formed from the dissociative adsorption of molecular oxygen. The local work function on the Pt surface changes from the value for clean $\mathrm{Pt}$ when either $\mathrm{CO}$ or $\mathrm{O}$ is adsorbed and a contrast in PEEM is originated. By using a deuterium discharge lamp for sample illumination, the authors 
observed in PEEM the formation of spiral propagation fronts across the $\operatorname{Pt}(100)$ and $\operatorname{Pt}(110)$ surfaces due to changes in the local surface coverage, as can be seen in Fig. 3. In this case, the time dependence of the propagation front evolution is clear from the increase of the dark area visible in the series of snapshots. Based on PEEM measurements the authors have also determined that, depending on the experimental conditions, a transition from regular oscillations to chaos is possible. These results nicely illustrate how PEEM can contribute to understand the way chemical reactions can occur on surfaces that are relevant in the field of catalysis.

LEEM/PEEM instruments can take advantage of the tunability (of photon energy and polarizability) and the high brilliance of nowadays state of the art synchrotron light sources for performing spectroscopic measurements by means of X-ray absorption spectroscopy (XAS), X-ray photoemission spectroscopy (XPS) and X-ray photoemission electron microscopy (XPEEM). The strength of this approach is that the combination of spectroscopic information obtained with these techniques with structural information obtained with
LEEM/LEED enables a thorough physicochemical characterization of a given material (see e.g., [20, 25]). However, it is at this point that the application of model surfaces becomes relevant; so correlations between structure and/or composition with chemical reactivity can be made. Many examples of this kind of characterization can be found in the literature for different types of model systems, ranging from bimetallic surfaces [26-28] to hybrid oxide-metallic systems [29-31].

During a chemical reaction the surface of the catalyst may change as a consequence of either the interaction of the reactants with the solid surface or due to the experimental conditions used (substrate temperature, reactant pressures, etc.). For instance, one of the most crucial steps in catalysis is the adsorption of the reactants. In multicomponent systems, like in multimetallic nanoparticles, each component may have different adsorptive properties towards one or even more reactants, characterized by significantly different energies of adsorption. As a consequence, a surface enrichment of one of the elements may occur over time under reactive
Fig. 3 PEEM observation of the propagation of a spiral wave during the catalytic oxidation of carbon monoxide on a $\mathrm{Pt}(110)$ surface under reaction conditions, showing its continuous growth. The ellipticity of the spiral is a consequence of the anisotropy of the surface diffusion coefficient of the reactants. Width of each image is $0.2 \mathrm{~mm}$. Sample temperature during acquisition was $434 \mathrm{~K}$. Reactants partial pressures were $\mathrm{p}_{\mathrm{CO}}=2.8 \times 10^{-5}$ mbar and $\mathrm{pO}_{2}$ $=3.0 \times 10^{-4}$ mbar. $\mathrm{t}=0,10,21$, 39, 56, and 74 s. Reprinted with permission from reference [23]

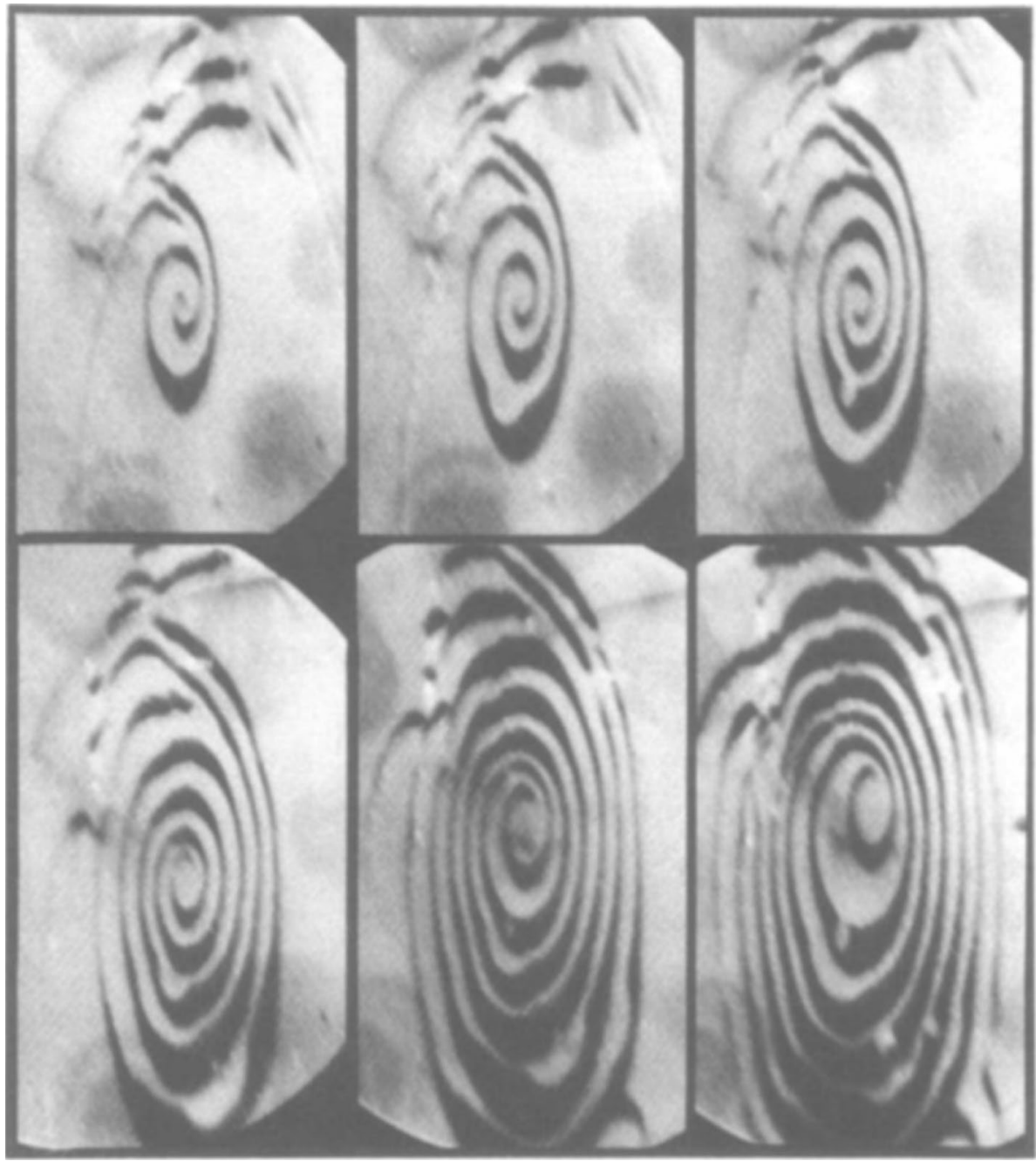


conditions, thus changing the original ability of the material to conduct the chemical reaction in the most efficient and low energy demanding path [32].

For instance, cerium oxide and vanadium oxide are important in catalysis and different model systems have been created in both cases to tackle fundamental questions [33-40]. On one side, $\mathrm{CeO}_{x}$ has the ability of easily interconvert the oxidation state of the $\mathrm{Ce}^{\mathrm{n}+}$ centres, making the material an interesting candidate as a catalyst for hydrocarbon oxidation reactions. Also, due to the highly oxidizing potential, $\mathrm{CeO}_{2}$ has been used successfully in automobile exhaust system to reduce the emissions of nitrogen oxides and carbon monoxide. Also, ceria has proven to be an interesting material for solid oxide fuel cells due to its good permeability towards oxygen at temperatures lower than those used for other oxides like zirconia. In connection with this, ceria has also proven to be quite active for the water gas shift reaction. For this reason, many researchers have focused in the last years on the development and study of model system that could eventually allow a comprehensive understanding of fundamental questions regarding the surface changes that take place under operando conditions and the interaction between the different components of the material. In this respect, a substantial contribution has been made by LEEM/ PEEM studies in the last years [31, 37, 38, 41, 42].

For instance, nanostructured gold supported on ceria is an important model system for the water shift reaction. However, it is not well understood yet what is the chemical state of the Au centres participating in the reaction. In order to gain some insight into this issue, Grinter and collaborators [26] used the combined LEEM/XPEEM approach and got indications of a charge transfer from Au nano-islands to the oxide support, and depending on the oxidation state of $\mathrm{Ce}^{\mathrm{n}+}$ within the oxide matrix, different Au species can be found, with $\mathrm{Au}-\mathrm{Ce}$ alloying being one of the possible scenario. A set of selected results reproduced from reference [26] is shown in Fig. 4.

By using local spectroscopy, these authors were able to follow the changes in the surroundings of the active parts of the model catalyst. For instance, Fig. 4 shows how $\mathrm{Au}$ changes its oxidation state when changes in the oxidation state of the ceria islands occur. As can be seen from the XPS spectra of $\mathrm{Au} 4 \mathrm{f}_{7 / 2}$ core line, the oxidation state of $\mathrm{Au}$ on direct contact with the $\mathrm{Rh}(111)$ surface does not
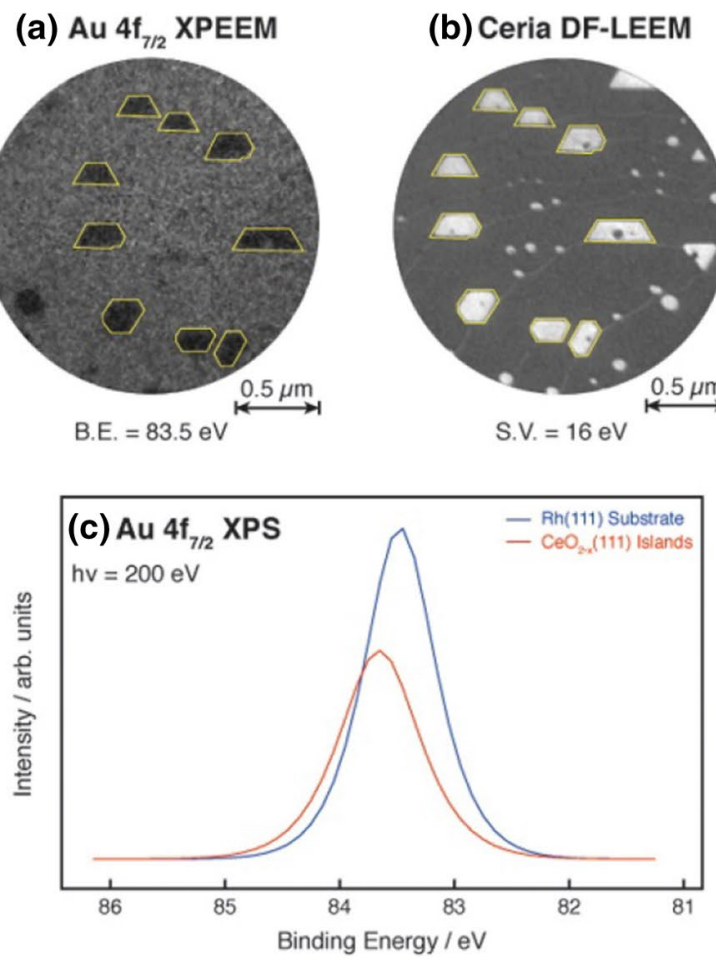

Fig. 4 XPEEM/LEEM and local XPS of $0.1 \mathrm{ML}$ of $\mathrm{Au}$ on $\mathrm{CeO}_{1.67}(111) / \mathrm{Rh}(111)$ showing the effects of beam-induced reduction of the ceria: a-c obtained in a background of $5 \times 10^{-7}$ mbar of $\mathrm{O}_{2}$ and d-f obtained in UHV. a Energy-filtered XPEEM at a B.E. of $83.5 \mathrm{eV}$ (Au $4 \mathrm{f}_{7 / 2}$ maximum on the $\mathrm{Rh}(111)$ substrate). b Dark-field LEEM $(\mathrm{S} . \mathrm{V} .=16 \mathrm{eV})$ from the ceria showing the film structure and island locations. c Au $4 \mathrm{f}_{7 / 2}$ XPS derived from energy-filtered XPEEM; the spectra obtained from the substrate and the ceria islands are shown
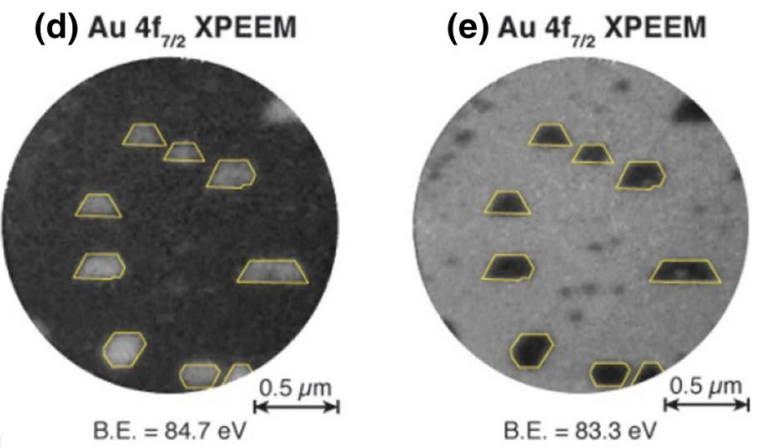

Reduce film (pump out $\mathrm{O}_{2}$ )

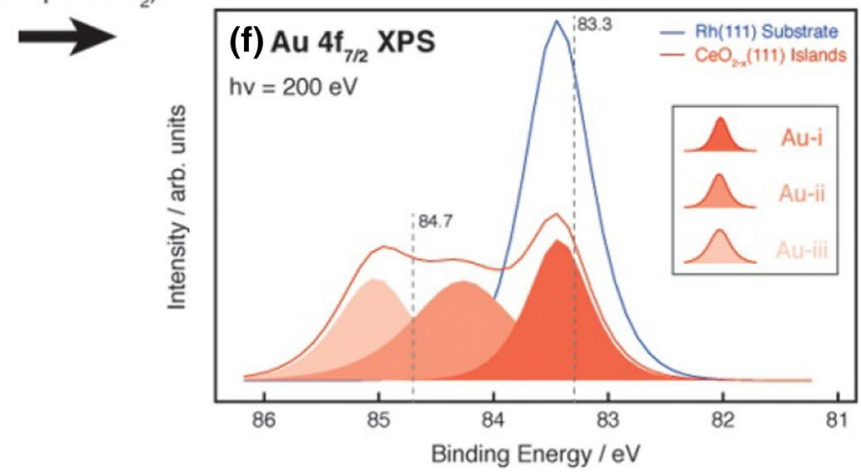

in blue and red, respectively. d Energy-filtered XPEEM at a B.E. of $84.7 \mathrm{eV}$. e Energy-filtered XPEEM at a B.E. of $83.3 \mathrm{eV}$. f Au $4 \mathrm{f}_{7 / 2}$ XPS derived from energy-filtered XPEEM; the spectra obtained from the substrate and the ceria islands are shown in blue and red, respectively. The largest ceria islands have been highlighted in green in all of the images as a visual aid $(\mathrm{h} \nu=200 \mathrm{eV}, \mathrm{FOV}=2 \mu \mathrm{m})$. Reprinted with permission from reference [26]. Copyright (2014) American Chemical Society 
change after the removal of molecular oxygen in the analysis chamber. However, in the case of Au present on top of the $\mathrm{CeO}_{2-\mathrm{x}}(111)$ reduced islands new components emerge at higher binding energy in XPS, being attributed by the authors to the formation of a Au-Ce alloy that happens only when the topmost layers of ceria islands are reduced.

Because, as stated before, one of the stronger points of the application of cerium oxide as a catalyst constituent is its ability to change oxidation state in a reversible way, their results are of great value in the sense that they prove and establish a framework to understand and assess the dynamics of the catalysis phenomena. Also, the authors could detect a spill over process between the $\mathrm{CeO}_{\mathrm{x}}$ species and the metallic substrate using the $\mathrm{CeOx} / \mathrm{Rh}(111)$ model system, giving thus some insight how the reversibility in the oxidation state of Ce centres happens. They demonstrated that the metallic substrate in this case has an important role in providing the necessary oxygen to re-oxidize and regenerate the oxide film [2].

On the other hand, the vanadium oxide system, in the same way as ceria based catalysts, has proven to be very active materials to catalyse oxidation reactions in industry [43]. Common applications include the oxidation of sulphur oxides for the production of sulfuric acid and in the production of phthalic anhydride, the last one being an important compound in the industry of plastics. Also, alternative application can be found in the field of oxidative dehydrogenation reactions $[44,45]$. Due to its wide applicability, it is important to rationally understand the working principle of the catalyst under different experimental conditions. In this sense, Lovis et al. [29, 30, 46] have used LEEM and PEEM to address the properties of a $\mathrm{V}_{\mathrm{x}} \mathrm{O}_{\mathrm{y}} / \mathrm{Rh}(111)$ model system, establishing a representative example of what can be done and what kind of information can be extracted from a model system with this approach. For instance, their studies were focused on the dynamic changes that occur on the oxide phase when the model catalyst is exposed to reaction condition, in this case being the hydrogen oxidation reaction. Some selected results extracted from reference [46] are shown in Fig. 5.

For instance, the authors were able to determine that when the model surface is exposed to $\mathrm{H}_{2}+\mathrm{O}_{2}$ mixtures, the surfaces restructures undergoing a pattern formation with alternating covered and uncovered areas on $\mathrm{Rh}(111)$, as can be clearly seen from the PEEM images shown in Fig. 5. The process has proven to be reversible and dependent on the total pressure of the reactants, with long transient times due to the low mobility of the oxide phase. The importance of this finding is that it directly shows the dynamic character of the surface, and although in this case is a two-phase material (oxide-metal interface), it may well happen in other systems (see e.g. [27, 47]). In catalysis, the structure and material composition of the surface is of fundamental importance for the chemical processes occurring during a catalytic reaction and in this respect, changes in the structure and/or composition of the surface might lead not only to changes in the kinetics of the chemical reaction but also on the selectivity of the catalyst.

A final important aspect where PEEM can offer significant help to answer fundamental questions is the local study of chemical reaction on a given substrate. In principle, one of the mechanisms of contrast formation in these techniques is the local variation of surface work function. This variation may arrive due to differences in composition in multicomponent systems, but also can originate when adsorbates are present on the surface. As a consequence, covered and uncovered regions on the surface of model catalysts or even regions having different adsorbates can exhibit different contrasts based on the differences in the local work function, making the surface chemical properties of the material visible in some way. On one side, it is well known that not all sites on a surface offer the same energetics for adsorption for
Fig. 5 PEEM images showing the response of the $\mathrm{V}$-oxide pattern to a continuous variation of $\mathrm{p}\left(\mathrm{H}_{2}\right)$ at $825 \mathrm{~K}$ and $\mathrm{p}\left(\mathrm{O}_{2}\right)=2.5 \times 10^{-6}$ mbar. $\mathrm{p}\left(\mathrm{H}_{2}\right)$ was cycled with a period of roughly $1 \mathrm{~min}$ : increase to $1.1 \times 10^{-6}$ mbar in images (a-d), then decrease to 0 in $(\mathbf{d}-\mathbf{g})$. The dashed lines indicate sharp changes in the state of the surface. (a-g) were successively recorded: with $\mathbf{a}$ at $\mathrm{t}=0$, b directly before it changes to $\mathbf{c}$ at $\mathrm{t}=50 \mathrm{~s}, \mathbf{d} \mathrm{t}=30 \mathrm{~s}$, e directly before it changes to $\mathbf{f}$ at $\mathrm{t}=50 \mathrm{~s}$, g at $\mathrm{t}=65 \mathrm{~s}$. Reprinted with permission from reference [46]

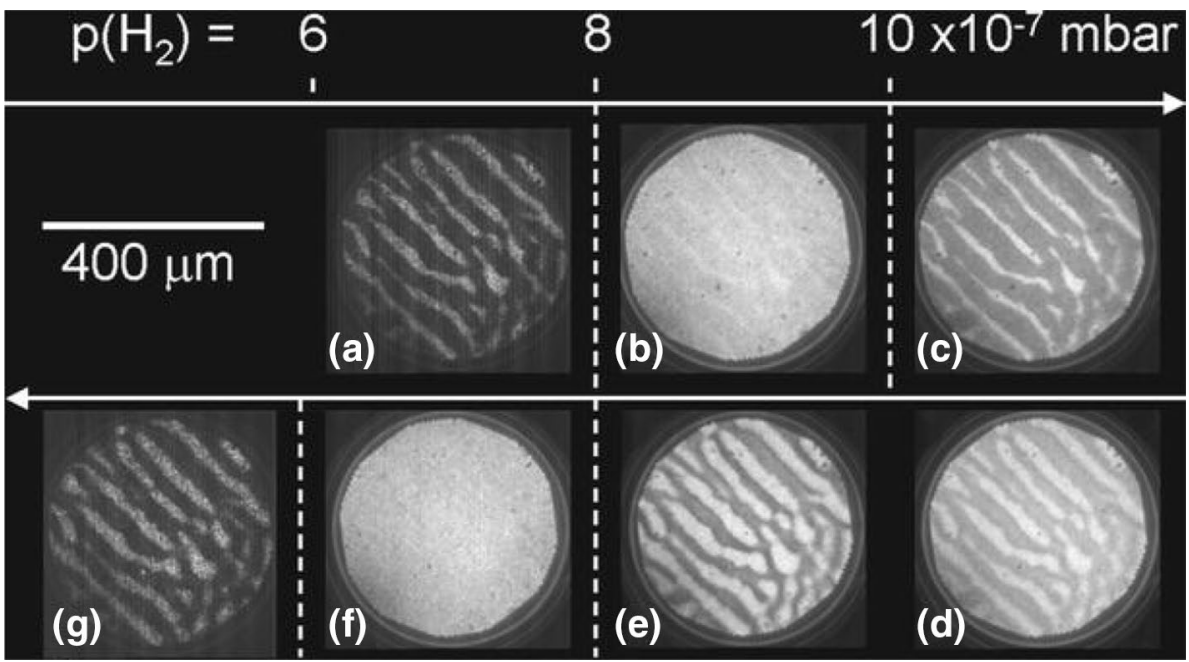


a given molecule. For instance, under-coordinated sites like atoms at step edges or kinks are more reactive (i.e. greater heat of adsorption) compared to atoms highly coordinated in terraces [10]. This is one of the reasons why surface sensitive reactions strongly depend on the local structure of the surface where the catalytic process occurs, with the dependence not only being reflected on the kinetics but also on the product distribution or selectivity. Consequently, photoemission electron microscopy turns out to be a valuable tool to image chemical reactions on model surfaces with catalytic activity and to address the influences of surface defects on the chemical reaction.

In the past decades, studies regarding the $\mathrm{CO}$ oxidation on different substrates using PEEM have been conducted [24, 48-51]. The nature of the pattern formation under well determined experimental conditions have been the main object of study. However, one important aspect than can also be addressed by using PEEM on surfaces under reaction conditions is the local kinetics of the reaction and how the surface structure can influence the reaction fronts under defined temperature and reactant pressure conditions (see e.g. [52]). At this point it is important to point out that the $\mathrm{CO}$ oxidation reaction is a chemical reaction that gives us some hints on the active sites on model catalysts, thus allowing the understating of how other more complex chemical reactions can proceed on model system surfaces. Moreover, in many important processes carbon monoxide is an intermediary specie that can undermine the activity of the catalyst by poisoning it, as in noble metal based materials [53]. In the context of local kinetics determination, the application of PEEM on the study of chemical reaction fronts on more complex model catalysts could shed some light on the changes in mechanism due to changes in either composition or structure of the material. For instance, the LEEM and PEEM have been used to study the intercalation of reactive gases such as $\mathrm{CO}$ and also chemical reactions under a graphene layer supported on a $\operatorname{Pt}(111)$ surface [54]. In this case, the combination of microscopy with diffraction and spectroscopy allowed to estimate the reaction rate and to determine the relevance of step edges as important surface sites for the reaction. Although the authors do not present a comparison with the oxidation rates on the bare Pt (111) surface, this could help the understanding of the effect of confinement on chemical reactions, a situation that could be found in very important materials in catalysis such as zeolites.

Regarding the specific case of oxides as model systems for catalysis, silica thin films have been prepared and well characterized in the Chemical Physics Department of the Fritz Haber Institute in Berlin. The importance of the $\mathrm{SiO}_{2} /$ $\mathrm{TM}(\mathrm{hkl})$ system relies on the fact that it constitutes a model system that can be used to study fundamental properties of applied systems with a great significance in the industry, such as zeolites (aluminium silicates) and the Phillips catalyst (chromium doped silica).

Experimental techniques with the main focus on structure determination (LEED, STM) have shown that silica thin film can be grown basically in two modes on different transition metal substrates, namely: monolayer and bilayer $[55,56]$. For a silica bilayer film, two different structures can be produced depending on the experimental conditions, namely: crystalline and amorphous [57]. The use of LEEM in this case can contribute to the understanding of how the transformation between the two phases occurs by following the process in real time either in the microscopy or diffraction mode. Another important issue is the thermal stability of the silica thin film. For instance, it is extremely important to determine the stability of the silica thin film under reaction conditions or at temperatures at which the chemical processes will be occurring on silica model system. Using LEEM and PEEM Klemm et al. [58] have investigated the thermal stability of such films in $\mathrm{O}_{2}$ atmosphere and found that at temperatures above $1100 \mathrm{~K}$ the silica films undergoes a de-wetting process with the consequent loss of material and the creation of mesoscopic holes that expose the $\mathrm{Ru}(0001)$ surface. This observation can be clearly seen when comparing Ru 3d and Si 2p XPEEM pictures with the LEEM picture presented on Fig. 6.

From the two states possible for the $\mathrm{SiO}_{2}$ bilayer, the vitreous phase seems to be the most promising so far in terms of the permeability to gases due to the presence of pores having more than 6 members [59]. In the case of the $\mathrm{SiO}_{2} /$ $\mathrm{Ru}(0001)$ system, it has been reported the possibility of intercalating simple molecules such as $\mathrm{CO}$ and $\mathrm{D}_{2}$ under the amorphous film, that means into the confined space enclosed between the silica sheet and the $\mathrm{Ru}(0001)$ surface, under well-defined experimental conditions. By using infrared reflection absorption spectroscopy (IRAS) Emmez et al. have shown by means of temperature programmed desorption (TPD) experiments that the silica bilayer acts as a barrier for $\mathrm{CO}$ and $\mathrm{D}_{2}$ to diffuse away from the Ru surface once they desorb [60]. In this sense, the thin silicon oxide film behave as a membrane that could, in principle, act as a size selective barrier ("molecular sieve") for diffusion of molecules being consumed or produced on the transition metal surface underneath during a chemical reaction, as illustrated in the scheme shown in Fig. 7.

An important point that has not yet been addressed for the intercalation gases in the $\mathrm{SiO}_{2} / \mathrm{Ru}(0001)$ system is the influence of mesoscopic holes such as those shown in Fig. 6 that can be created during the preparation procedure of the bilayer. When compared to the pores naturally present in the vitreous phase, these mesoscopic holes could as well influence the dynamics and energetics of the diffusion of gases through the oxide film and also modify the maximum attainable coverage possible 

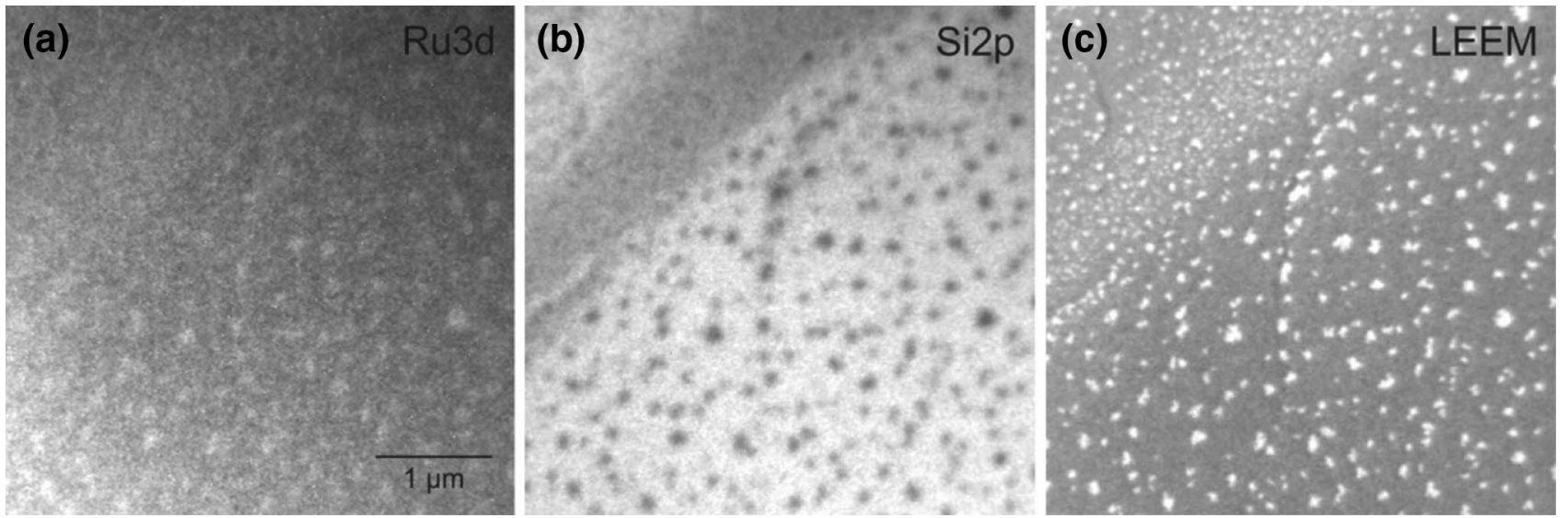

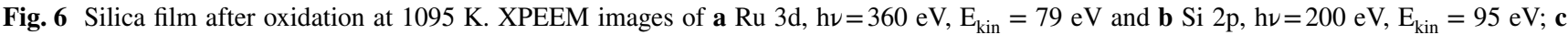
LEEM image at $\mathrm{E}_{\mathrm{kin}}=79 \mathrm{eV}$. Reprinted with permission from reference [58]

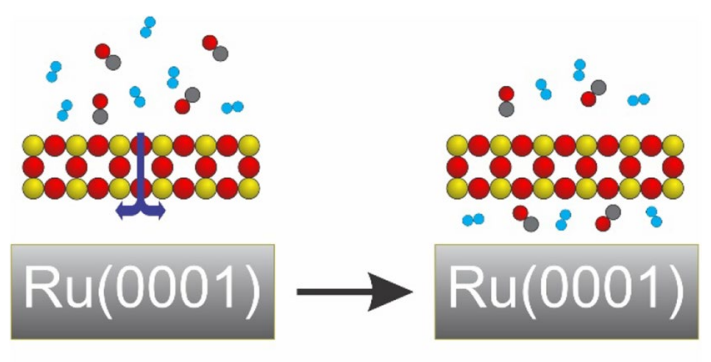

- Hydrogen O Silicon Oxygen C Carbon

Fig. 7 Sphere model of the $\mathrm{SiO}_{2} / \mathrm{Ru}(0001)$ system showing how gases can intercalate through the bilayer and reach the Ru surface

for reactants. In either case, these seem to be important parameters that might influence the kinetics of chemical reactions occurring in the space confined between the silica film and the metal support.

Another important point in the $\mathrm{SiO}_{2} / \mathrm{Ru}(0001)$ system is that, unlike bare $\mathrm{Ru}(0001)$ [61] where the rutile $\mathrm{RuO}_{2}$ phase can be formed, exposing a silica-covered Ru surface to similar oxidizing conditions reveals a suppression of the oxidation extent [62]. In this sense, these results clearly show that confinement has a negative effect on the oxidation reaction occurring at the Ru surface. This issue can be further explored for other relatively simple chemical reactions in order to gain insight conceptually on the effect of confinement on the kinetics of the reaction. Just in this aspect LEEM and PEEM can contribute to this issue, by allowing the monitoring of the chemical reaction in real time and enabling the activation energy for simple processes that can be compared with values obtained for bare substrates.

\section{Future Perspectives}

In the previous section we discussed what we think are representative cases illustrating the strength of techniques such as LEEM and PEEM, in comparison with other traditional surface science methods. Despite the significance of the results discussed, it is important to recognize that new developments on these techniques might eventually lead to a deeper understanding of well-known problems that cannot, at the moment, be studied with them.

One important issue common to most oxide-based systems has to be considered, when working with electronbased techniques such as LEEM: thin oxide films can suffer from e-beam induced damages while performing measurements. The degree of beam damage depends obviously on the chemical nature of the oxide, but is certainly an important issue that needs to be taken into account when performing in situ and in operando experiments, where the presence of gases (reactants) can trigger sample degradation, or when long acquisition times are required. In this regard, the successful correction of both, chromatic and spherical aberrations, in modern LEEM instruments have allowed the use of less demanding conditions to obtain images in terms of electron dosing on the samples and faster acquisition time $[19,63,64]$. The advantage of double aberration correction can be reflected on two different aspects. First of all, this correction enables a higher spatial resolution and the advantages in this sense are quite straightforward. On the other hand, the elimination of chromatic and spherical aberrations facilitates the use of larger acceptance angles at the sample and with this, the transmission can be enhanced by up to two orders of magnitude, making it advantageous not only for avoiding sample degradation but also for real time measurements by decreasing the acquisition time. 
Another aspect that is being considered by some companies $[65,66]$ responsible for the development of commercial LEEM/PEEM instruments is the possibility of performing measurements in the mbar range, in the so-called near ambient pressure regime, with LEEM instruments (NAP-LEEM). New processes that before were kinetically hindered in the $10^{-10}-10^{-6}$ mbar range can now be explored under these mbar conditions that are in principle much closer to real operating conditions. The set-ups are not commercially available yet, however the developers of ELMITEC have already successfully tested a NAP-LEEM system for imaging a $\mathrm{Si}(100)$ etched-patterned surface exposed to $0.1 \mathrm{mbar}$ of air, as can be seen in Fig. 8 .

This new operating mode of a LEEM and possibly PEEM instrument can potentially have a big impact on the type of issues that can be accessed with these techniques. For instance, Tao et al. have shown using near ambient pressure scanning tunnelling microscopy (NAP-STM) that vicinal surfaces of Pt can show a reconstruction when exposed to $\mathrm{CO}$ pressures in the mbar range, giving as a result a break up on the stepped nature of the surface [67]. Although this was studied by the authors on single crystalline surfaces, their results raise an important question about the structure of the
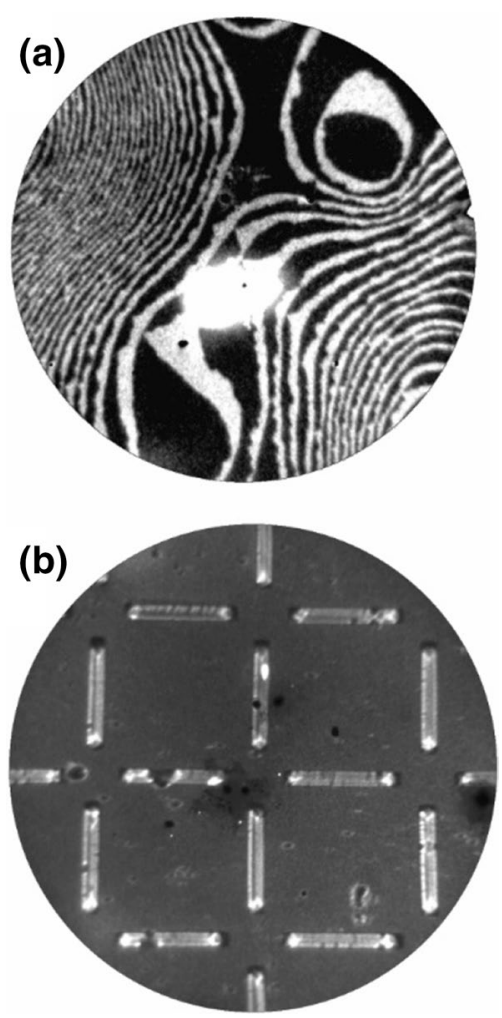

Fig. 8 LEEM (a) and PEEM (b) images of a Si(100) patterned substrate acquired in $1 \times 10^{-9}$ mbar and $0.1 \mathrm{mbar}$ air, respectively. Starting voltage used for image acquisition are 4.45 and $4 \mathrm{eV}$, respectively. Fields of view of images $\mathbf{a}$ and $\mathbf{b}$ are 1.6 and $90 \mu \mathrm{m}$. Images are a courtesy of ELMITEC Elektronenmikroskopie GmbH surfaces on model catalysts under reaction conditions; i.e., model surfaces might undergo the same kind of structural transformation when exposed to more realistic conditions in terms of reactant pressures during in situ and real time experiments.

Another important development in LEEM/PEEM instruments in the catalysis community is related to its applicability in the study of relevant systems in the area of electrocatalysis. This is an important field that has also received the attention of many researchers, especially in the topic of fuel cell for the generation of clean energy. Many model systems in this area are composed by metal nanoparticles (bimetallic or trimetallic) supported on either metallic single crystals or well-ordered oxide surfaces. It has been reported in the literature the possibility of using PEEM for the in situ study of electrochemically induced oxygen spill over on Pt/ YSZ (YSZ: yttria stabilized zirconia) [68]. In this case, the authors used a three-electrode cell with a solid electrolyte and were able to indirectly follow in real time the changes on a Pt surface due to the reaction of a specie generated electrochemically. On the other hand, it has been also reported the possibility of measuring with PEEM a liquid-solid interphase by using a graphene-capped microchannel array [69]. The idea behind this approach is to isolate the imaging components of LEEM/PEEM microscopes from the high pressure environment at which the samples are submitted in order to avoid technical problems with the high voltages typically used $(15-20 \mathrm{kV})$. In this sense, graphene-based windows have proven to be quite robust, in terms of stability towards reactive gases and high pressures, and transparent to electrons [70-72]. In the case of the microarray, the principle is the same, i.e., the liquid phase is trapped in the pores of porous materials that are covered with graphene. With this experimental array, the authors were able to study the waterlgraphene interface with PEEM for the first time. At the moment, the stage of developments is primordial, but might open the door for new ideas for the application of LEEM and PEEM as potential tools to address questions in fields like electrocatalysis or even corrosion.

Finally, another important aspect of the application of LEEM and PEEM is the possibility of performing ultra-fast measurements to follow structural processes or reactions at much shorter time scale (sub nanosecond regime), with the potential capability of identifying reactions intermediates and allowing correlations with the surface structure [73]. The approach of time-resolved measurements with high time resolution in the picosecond range with PEEM has been already reported for the study of magnetic properties of certain materials $[74,75]$ and, more recently, for the study of plasmon propagation on metallic surfaces [76]. In general, the approach for the detection of ultrafast processes is based on the pump and probe method. In this method two signals are used in consecutive pulses, first to bring the system in an 
excited state (pump) and then to detect and follow the decay of such excited state (probe). The time interval between the pulses $(\Delta t)$ will determine ultimately the time scale of the observed processes (fs or ps). In this sense, the nature of the pulses can have different origin, depending on the properties that want to be studied (for more details see [77]), making also use of the possibilities offered by third generation synchrotron storage rings. For example, magnetic pulses followed by light pulses can give some information on the surface domain reorganization dynamics in magnetic systems by using XMCD [75]. A further example is the two-photon photoemission spectroscopy (2PPES) which is a powerful tool to assess different aspects of electron dynamics on metals and semiconductors. Here, two different light pulses are used with different energy. The first pulse promotes electrons into an excited unoccupied state just between Fermi and vacuum level and the second pulse excites the electrons above the vacuum level, emitting them and making it possible to analyse the intermediate state. A regular PEEM instrument could make use of the 2PPE approach giving as a result basically the same information as in the spectroscopy mode (2PPES) but with spatial resolution in nanometer range. This advantage might become very useful to assess, for example, the structural dependence of hot electrons dynamics on heterogeneous surfaces relevant in catalysis. Interestingly, the influence of hot electrons on the activation or modification of surface reactions or physical processes in some molecular systems has been addressed in the literature [78-80]. For instance, it has been suggested that hot electrons can influence processes that might represent important steps in relevant catalytic processes, such as surface diffusion, cis-trans conversion, adsorption/desorption and activation in different heterogeneous systems (see [80] and references therein). The fact is that morphology-electronic structure relationships are not so well understood regarding these effects in the microscopic scale and, it is in this aspect that state-of-the-art high time resolution PEEM can contribute to a better fundamental understanding by combining spectroscopic information with the spatially resolved information on metal-oxides model systems.

\section{Concluding Remarks}

We have presented some selected examples that illustrate how low energy electron microscopy and photoemission electron microscopy can contribute to the understanding of fundamental properties of model systems relevant in the area of catalysis. All examples show the advantage of combining spectroscopic information with spatial resolution on heterogeneous surface, thus making possible to use a real-time and in situ approach. However, a lot more could be done in the future to take the application of PEEM and LEEM to a higher level where new questions in fundamental aspects of catalysis can be addressed by using new experimental approaches that would eventually allow a deeper understanding and eventually aid the rational design of real catalytic materials.

Acknowledgements Open access funding provided by Max Planck Society. We thank H.-J. Freund for fruitful discussions and his encouragement for this perspective paper. We thank also $\mathrm{H}$. Marchetto from Elmitec Elektronenmikroskopie $\mathrm{GmbH}$ for his collaboration and fruitful discussion regarding new instrumental development.

Open Access This article is distributed under the terms of the Creative Commons Attribution 4.0 International License (http://creativecommons.org/licenses/by/4.0/), which permits unrestricted use, distribution, and reproduction in any medium, provided you give appropriate credit to the original author(s) and the source, provide a link to the Creative Commons license, and indicate if changes were made.

\section{References}

1. Schlögl R (2015) Angew Chem Int Ed Engl 54:3465

2. Grinter DC, Muryn C, Sala A, Yim CM, Pang CL, Menteş TO, Locatelli A, Thornton G (2016) J Phys Chem C 120:11037

3. Over H (2012) Chem Rev 112:3356

4. Shao M, Chang Q, Dodelet J-P, Chenitz R (2016) Chem Rev 116:3594

5. Schwach P, Pan X, Bao X (2017) Chem Rev 117:8497

6. Lloyd L (2011) Handbook of industrial catalysts. Springer, Berlin

7. Schmal M (2016) Heterogeneous catalysis and its industrial applications. Springer, Berlin

8. Ertl G (2010) Reactions at solid surfaces. Wiley, Hoboken

9. Zaera F (2005) Chem Rec 5:133

10. Somorjai GA, McCrea KR, Zhu J (2002) Top Catal 18:157

11. Védrine JC (2014) Appl Catal A 474:40

12. Baraldi A, Bianchettin L, de Gironcoli S, Vesselli E, Lizzit S, Petaccia L, Comelli G, Rosei R (2011) J Phys Chem C 115:3378

13. Greeley JP (2012) Science 336:810

14. Wang AQ, Liu XY, Mou CY, Zhang T (2013) J Catal 308:258

15. Brüche E (1933) Z Phys 86:448

16. Bauer E (2012) J Electron Spectrosc Relat Phenom 185:314

17. Bauer E, (1994) Rep Prog Phys 57:895.

18. Bauer E (2014) Surface microscopy with low energy electrons. Springer, New York

19. Schmidt T, Marchetto H, Lévesque PL, Groh U, Maier F, Preikszas D, Hartel P, Spehr R, Lilienkamp G, Engel W, Fink R, Bauer E, Rose H, Umbach E, Freund HJ (2010) Ultramicroscopy 110:1358

20. Schmidt T, Groh U, Fink R, Umbach E, Schaff O, Engel W, Richter B, Kuhlenbeck H, Schlögl R, Freund H-J, Bradshaw AM, Preikszas D, Hartel P, Spehr R, Rose H, Lilienkamp G, Bauer E, Benner G, (2002) Surf Rev Lett 9:223

21. Tromp RM (2012) Ultramicroscopy 120:73

22. Bauer E (2012) Ultramicroscopy 119:18

23. Jakubith S, Rotermund HH, Engel W, Vonoertzen A, Ertl G (1990) Phys Rev Lett 65:3013

24. Rotermund HH, Engel W, Kordesch M, Ertl G (1990) Nature 343:355

25. Schmidt T, Heun S, Slezak J, Diaz J, Prince KC, Lilienkamp G, Bauer E, (1998) Surf Rev Lett 05:1287.

26. Grinter DC, Muryn C, Santos B, Shaw BJ, Mentes TO, Locatelli A, Thornton G (2014) J Phys Chem C 118:19194

27. Locatelli A, Heun S, Kiskinova M (2004) Surf Sci 566-568:1130 
28. Flege JI, Lachnitt J, Mazur D, Sutter P, Falta J (2016) Phys Chem Chem Phys 18:213

29. Lovis F, Imbihl R (2011) J Phys Chem C 115:19141

30. Lovis F, Hesse M, Locatelli A, Menteş TO, Niño MÁ, Lilienkamp G, Borkenhagen B, Imbihl R (2011) J Phys Chem C 115:19149

31. Höcker J, Menteş TO, Sala A, Locatelli A, Schmidt T, Falta J, Senanayake SD, Flege JI (2015) Adv Mater Interfaces 2:1500314

32. Zafeiratos S, Piccinin S, Teschner D (2012) Catal Sci Technol 2:1787

33. Pan Y, Nilius N, Stiehler C, Freund H-J, Goniakowski J, Noguera C (2014) Adv Mater Interfaces 1:1400404

34. Romanyshyn Y, Guimond S, Kuhlenbeck H, Kaya S, Blum RP, Niehus H, Shaikhutdinov S, Simic-Milosevic V, Nilius N, Freund HJ, Ganduglia-Pirovano MV, Fortrie R, Dobler J, Sauer J (2008) Top Catal 50:106

35. Guimond S, Abu Haija M, Kaya S, Lu J, Weissenrieder J, Shaikhutdinov S, Kuhlenbeck H, Freund HJ, Dobler J, Sauer J (2006) Top Catal 38:117

36. Ganduglia-Pirovano MV, Popa C, Sauer J, Abbott H, Uhl A, Baron M, Stacchiola D, Bondarchuk O, Shaikhutdinov S, Freund H-J (2010) J Am Chem Soc 132:2345

37. Grinter DC, Pang CL, Muryn CA, Maccherozzi F, Dhesi SS, Thornton G (2014) J Electron Spectrosc Relat Phenom 195:13

38. Grinter DC, Senanayake SD, Flege JI (2016) Appl Catal B 197:286

39. Göbke D, Romanyshyn Y, Guimond S, Sturm JM, Kuhlenbeck H, Döbler J, Reinhardt U, Ganduglia-Pirovano MV, Sauer J, Freund H-J (2009) Angew Chem Int Ed 48:3695

40. Abbott HL, Uhl A, Baron M, Lei Y, Meyer RJ, Stacchiola DJ, Bondarchuk O, Shaikhutdinov S, Freund HJ (2010) J Catal 272:82

41. Flege JI, Hocker J, Kaemena B, Mentes TO, Sala A, Locatelli A, Gangopadhyay S, Sadowski JT, Senanayake SD, Falta J (2016) Nanoscale 8:10849

42. Hocker J, Duchon T, Veltruska K, Matolin V, Falta J, Senanayake SD, Flege JI (2016) J Phys Chem C 120:4895

43. Thomas CL (1971)Catalytic processes and proven catalysts. Academic Press, New York, London

44. Bartholomew CH, Farrauto RJ, (2005) Catalytic oxidations of inorganic and organic compounds, fundamentals of industrial catalytic processes. Wiley, Hoboken p. 560

45. Wachs IE (2013) Dalton Trans 42:11762

46. Lovis F, Hesse M, Imbihl R (2010) Catal Lett 136:171

47. Schmidt T, Schaak A, Günther S, Ressel B, Bauer E, Imbihl R (2000) Chem Phys Lett 318:549

48. Mundschau M, Kordesch ME, Rausenberger B, Engel W, Bradshaw AM, Zeitler E (1990) Surf Sci 227:246

49. Rotermund HH (1999) J Electron Spectrosc Relat Phenom 98:41

50. Kelling S, Cerasari S, Rotermund HH, Ertl G, King DA (1998) Chem Phys Lett 293:325

51. Rotermund HH, Nettesheim S, Von Oertzen A, Ertl G (1992) Surf Sci 275:L645

52. Vogel D, Spiel C, Suchorski Y, Urich A, Schlögl R, Rupprechter G (2011) Surf Sci 605:1999

53. Argyle M, Bartholomew C, (2015) Catalysts 5:145

54. Mu R, Fu Q, Jin L, Yu L, Fang G, Tan D, Bao X (2012) Angew Chem Int Ed 51:4856
55. Yang B, Kaden WE, Yu X, Boscoboinik JA, Martynova Y, Lichtenstein L, Heyde M, Sterrer M, Wlodarczyk R, Sierka M, Sauer J, Shaikhutdinov S, Freund H-J (2012) Phys Chem Chem Phys 14:11344

56. Löffler D, Uhlrich JJ, Baron M, Yang B, Yu X, Lichtenstein L, Heinke L, Büchner C, Heyde M, Shaikhutdinov S, Freund H-J, Włodarczyk R, Sierka M, Sauer C (2010) Phys Rev Lett 105:146104

57. Heyde M, Shaikhutdinov S, Freund HJ (2012) Chem Phys Lett 550:1

58. Klemm HW, Peschel G, Madej E, Fuhrich A, Timm M, Menzel D, Schmidt T, Freund HJ (2016) Surf Sci 643:45

59. Büchner C, Liu L, Stuckenholz S, Burson KM, Lichtenstein L, Heyde M, Gao H-J, Freund H-J (2016) J Non-Cryst Solids 435:40

60. Emmez E, Yang B, Shaikhutdinov S, Freund HJ (2014) J Phys Chem C 118:29034

61. Flege JI, Sutter P, (2009) J Phys-Condens Matter 21:314018

62. Emmez E, Anibal Boscoboinik J, Tenney S, Sutter P, Shaikhutdinov S, Freund H-J (2016) Surf Sci 646:19

63. de la Figuera J, McCarty KF, (2013) Low-energy electron microscopy. In: Bracco G, Holst B (eds) Surface science techniques. Springer, Berlin, p 531t;/bib>

64. Schmidt T, Sala A, Marchetto H, Umbach E, Freund HJ (2013) Ultramicroscopy 126:23

65. Marchetto $\mathrm{H}$, Personal communication.

66. Schaff O, Breitschaft M, Kampen TU, Thissen A, Low energy electron microscopy at near ambient pressures.10th LEEM/PEEM workshopMonterey, California, p 87

67. Tao F, Dag S, Wang LW, Liu Z, Butcher DR, Bluhm H, Salmeron M, Somorjai GA (2010) Science 327:850

68. Luerssen B, Mutoro E, Fischer H, Gunther S, Imbihl R, Janek J (2006) Ang Chem-Int Ed 45:1473

69. Guo H, Strelcov E, Yulaev A, Wang J, Appathurai N, Urquhart S, Vinson J, Sahu S, Zwolak M, Kolmakov A (2017) Nano Lett 17:1034

70. Kraus J, Reichelt R, Gunther S, Gregoratti L, Amati M, Kiskinova M, Yulaev A, Vlassiouk I, Kolmakov A (2014) Nanoscale 6:14394

71. Kolmakov A, Gregoratti L, Kiskinova M, Günther S (2016) Top Catal 59:448

72. Kolmakov A, Dikin DA, Cote LJ, Huang J, Abyaneh MK, Amati M, Gregoratti L, Gunther S, Kiskinova M, (2011) Nat Nano 6:651

73. Locatelli A, Bauer E, (2008) J Phys-Condens Matter 20:093002

74. Schneider CM, Krasyuk A, Nepijko SA, Oelsner A, Schönhense G, (2006) J Magn Magn Mater 304:6

75. Neeb D, Krasyuk A, Oelsner A, Nepijko SA, Elmers HJ, Kuksov A, Schneider CM, Schönhense G (2005) J Phys Condens Matter 17:S1381

76. Gong Y, Joly AG, Hu D, El-Khoury PZ, Hess WP (2015) Nano Lett 15:3472

77. Schönhense G, Elmers HJ (2006) Surf Interface Anal 38:1578

78. Mukherjee S, Libisch F, Large N, Neumann O, Brown LV, Cheng J, Lassiter JB, Carter EA, Nordlander P, Halas NJ (2013) Nano Lett 13:240

79. Kim M, Lin M, Son J, Xu H, Nam J-M, (2017) Adva Opt Mater 1700004

80. Park JY, Baker LR, Somorjai GA (2015) Chem Rev 115:2781 